\title{
Rapidly Selective Growth of Nanoparticles by Electron-Beam and Optical Lithographies with Chemically Amplified Resists
}

\author{
H. L. Chen, ${ }^{\text {a,z }}$ Y. H. Chu, ${ }^{\text {b }}$ C. I. Kuo, ${ }^{\text {b }}$ F. K. Liu, ${ }^{\text {c** F. H. Ko, }}{ }^{\text {c,* }}$ and T. C. Chu ${ }^{b}$ \\ ${ }^{a}$ Department of Materials Science and Engineering, National Taiwan University, Taipei, Taiwan \\ ${ }^{b}$ National Tsing Hua University, Hsinchu, Taiwan \\ ${ }^{c}$ National Nano Device Laboratories, Hsinchu, Taiwan
}

\begin{abstract}
Rapidly and precisely selective growth of self-assembled nanoparticles using an electron-beam or optical exposure system with commercial chemically amplified resists was demonstrated. The required exposure dosage was less than $10 \mu \mathrm{C} / \mathrm{cm}^{2}$ for patterning chemically amplified resists. By immersing the patterned resist sample in the nanoparticle colloidal solution, nanoparticles were selectively self-assembled on the (3-aminopropyl)trimethoxysilane layer, which was partially covered by the patterned resist layer. The selective growth area can be performed from several hundred micrometers to sub-50 $\mathrm{nm}$. This method has great potential to be used for rapidly selective growth of various nanoparticles or nanomaterials.

(C) 2004 The Electrochemical Society. [DOI: 10.1149/1.1845051] All rights reserved.
\end{abstract}

Manuscript submitted July 14, 2004; revised manuscript received September 11, 2004. Available electronically December 16, 2004.

Nanometer particles have received much attention as potentially useful materials showing novel electronic, optical, magnetic, and thermal properties derived from the "quantum sized effect." 1 Therefore, selective growth of nanometer-sized materials is essential for biotechnology, optics, and semiconductor device fabrications. Many efforts have been made in selective growth of self-assembled monolayers (SAM). Atomic force microscopy (AFM) was used to locally oxidize the silicon surface and to perform spatially selective deposition of gold nanoparticles via specific chemical interaction. ${ }^{2,3}$ The conductive AFM tip can also be used to convert organosilane monolayer with terminal $-\mathrm{CH}_{3}$ groups to $-\mathrm{COOH}$. The $-\mathrm{COOH}$ patterns generated in monolayers are compatible with a wide variety of post-patterning chemical manipulations. ${ }^{4,5}$ Another approach is using high dosage of electron beam to create surface domains with different chemical properties that allow to selective deposit nanomaterials. In general, over $1000 \mu \mathrm{C} / \mathrm{cm}^{2}$ dosage of electron beam exposure is required. ${ }^{6}$ Recently, it was fond that by using low electron beam acceleration voltages one can reduce the required exposure dosage to less than $1000 \mu \mathrm{C} / \mathrm{cm}^{2} .7,8$ Similarly, exposing UV light source with long duration can also be used for selective growth of nanomaterials. ${ }^{9}$ However, all of these methods are low-throughput or difficult to combine with semiconductor processes. Because the process conditions are strongly dependent on the kind of nanomaterials, it is still a challenge to develop an effective way for selective growth of various nanomaterials by using these methods.

In the recent International Technology Roadmap for Semiconductor (ITRS), advanced lithographic techniques such as vacuum ultraviolet (VUV) and electron-beam-based lithographies would lead integrated circuit (IC) technologies to sub-70 nm generations. ${ }^{10}$ Chemically amplified resists (CAR) with high sensitivity have been widely used in deep ultraviolet (DUV) lithography and will continue to be used in next generation vacuum ultraviolet and electron beam based lithographies for high-throughput consideration. ${ }^{11}$ The chemical amplified resist is initiated by a couple of electrons or photons, and acid is generated in the resist film. The acid generator will react with the acid-labile group (attached to a polymer) within the resist film and lead to bond scission. The new molecule will generate a new acid, thus beginning a catalytic cycle. In general, an initial acid will produce 500 to 1000 chemical reactions. As a result, the applied dosage can be decreased and the throughput is enhanced. By using chemically amplified resists, the required exposure dosages in optical and electron beam exposure systems were generally less than $30 \mathrm{~mJ} / \mathrm{cm}^{2}$ and $20 \mu \mathrm{C} / \mathrm{cm}^{2}$, respectively. ${ }^{12}$ In this paper, selective

* Electrochemical Society Active Member.

z E-mail: hsuenlichen@ccms.ntu.edu.tw growth of self-assembled nanoparticles using electron-beam and optical exposure systems with commercial chemically amplified resists was demonstrated.

\section{Experimental}

All chemical materials were G. R. grade. Sodium citrate and hydrogen tetrachloroaurate $\left(\mathrm{HAuCl}_{4}\right)$ were purchased from Tokyo Chemical Industry. Colloidal gold solution was prepared by sodium citrate reduction of $\mathrm{HAuCl}_{4}$ under boiling conditions. ${ }^{13,14}$ The silicon oxide films were deposited on silicon wafers by employing a conventional high-density plasma chemical vapor deposition (HDPCVD) system. The (3-aminopropyl)trimethoxysilane (APTMS) layer, an adhesion agent for gold nanoparticles, was coated on a $\mathrm{SiO}_{2} / \mathrm{Si}$ substrate. ${ }^{15}$ Then the chemical amplified resist (DSE-1010, Dongjin) was coated on the APTMS layer for electron-beam exposure. The thickness of chemical amplified resist was measured by an ellipsometer (SE-5, Sopra). Electron-beam exposure was carried out in the Leica Weprint-200 shaped-beam stepper. The electron beam energy was $40 \mathrm{keV}$ with a beam size of $20 \mathrm{~nm}$. The exposure doses were changed from 3 to $20 \mu \mathrm{C} / \mathrm{cm}^{2}$. By increasing the exposure dose to $6.2 \mu \mathrm{C} / \mathrm{cm}^{2}$, these resists can be completely removed after developing with $2.38 \%$ tetramethylammonium hydroxide. After being immersed, the patterned resist sample into the gold nanoparticles colloidal solution for $10 \mathrm{~min}$, nanoparticles were self-assembled on the APTMS layer, which was partially covered by the patterned resist layer. Finally, ethyl alcohol was used to stripe the residual resists. Similarly, optical lithography (I-line i5 + stepper, Canon) was also used for nanomaterials patterning. Nanoparticles were observed by scanning electron microscopy (S-4000, Hitachi). The surface plasma resonance (SPR) absorption spectra of nanoparticles with and without selective growth processes were measured by a spectrometer (UV-2501PC, Shimadzu).

\section{Results and Discussion}

Figure 1a is the schematic description for selective growth of the self-assembled gold nanoparticles by electron beam or optical lithography. The APTMS layer, an adhesion agent for gold nanoparticles, was first coated on a $\mathrm{SiO}_{2} / \mathrm{Si}$ substrate. Then, the chemical amplified resist was coated on the APTMS layer for electron-beam exposure. Figure $1 \mathrm{~b}$ shows the sensitivity curve of the chemically amplified resist DSE-1010 for electron-beam exposure. By increasing the exposure dose to $6.2 \mu \mathrm{C} / \mathrm{cm}^{2}$, these resists can be completely removed after developing with tetramethylammonium hydroxide (TMAH). The high sensitivity and high contrast $(\gamma=19)$ of the resist are capable of ensuring high throughput and resolution in electron-beam lithography. After the sample was immersed in the gold nanoparticles colloidal solution for $10 \mathrm{~min}$ on a hot plate, nano- 


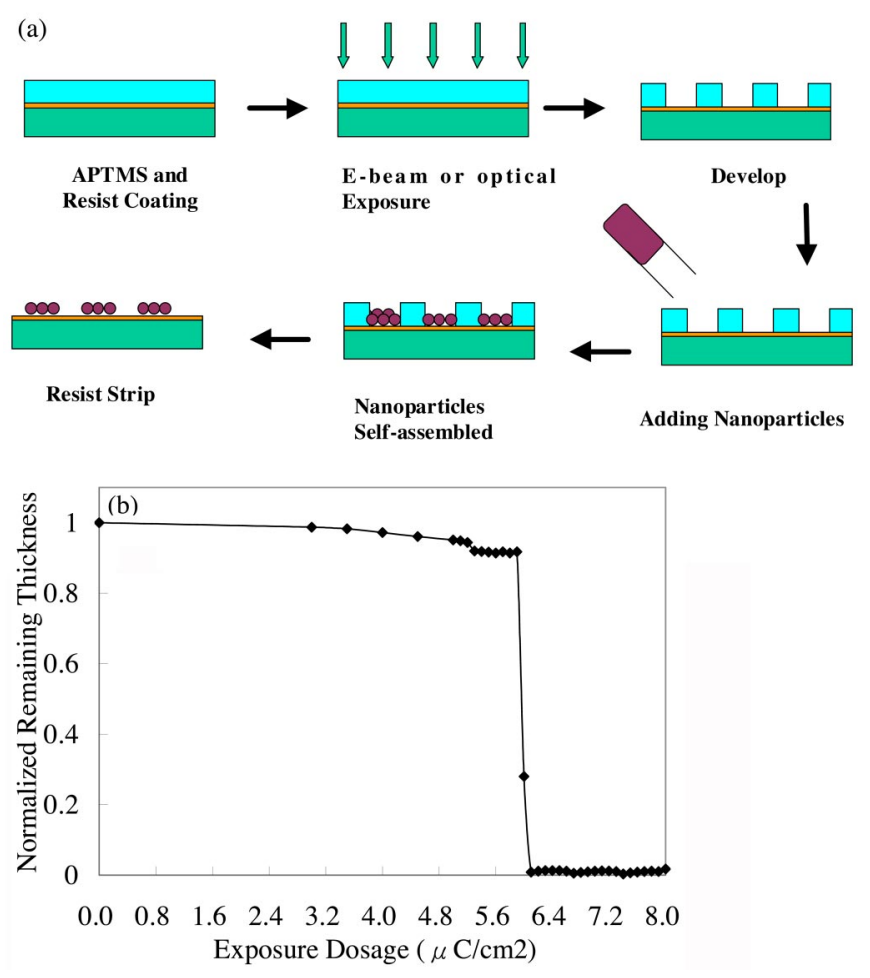

Figure 1. (a) Schematic diagram for selective growth of the self-assembled nanoparticles by electron beam or optical lithography. (b) The sensitivity curve of DSE 1010 chemical amplified resist at $40 \mathrm{keV}$ electron-beam exposure.

particles were self-assembled on the APTMS layer, as shown in Fig. 2. Finally, ethyl alcohol was used to stripe the residual resists. Similarly, optical lithography was also used for selective growth of nanomaterials. Using the optical stepper the throughput is generally larger than 100 wafers per hour.

Figure 2a shows the SEM image of dot array patterns of gold nanoparticles, which were patterned by optical lithography. The magnified image in Fig. 2b shows that nanoparticles were found uniformly dispersed in the pattern. Size-distribution and standard deviation (S.D.) of gold nanoparticles without selective growth pro-

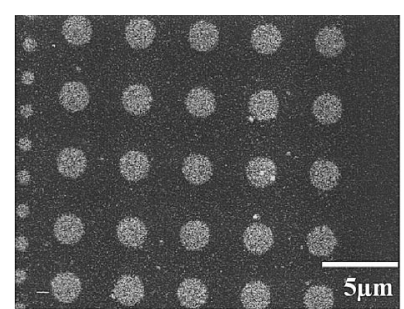

(a)

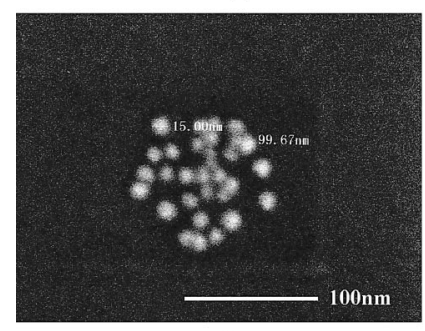

(c)

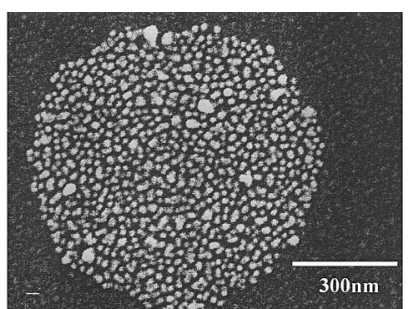

(b)

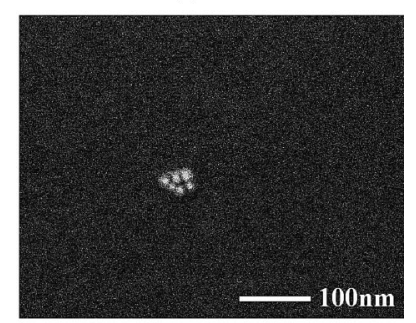

(d)
Figure 2. SEM images of gold nanoparticles selective growth by ( $a$ and $b$ ) optical lithography and (c and d) by electron beam lithography.
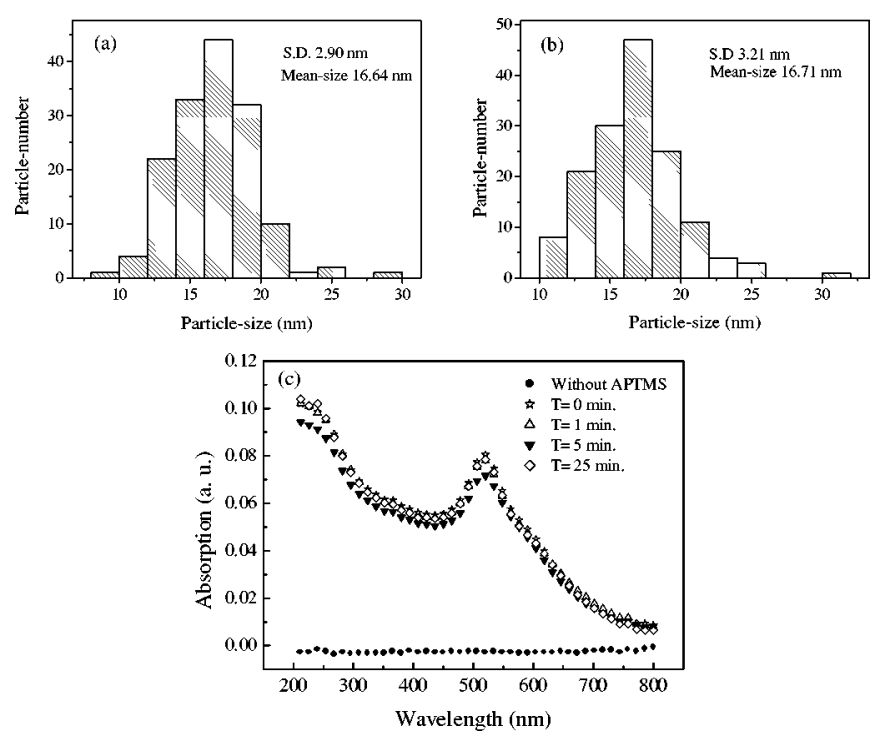

Figure 3. Size-distribution and S.D. of gold nanoparticles (a) without and (b) with selective growth processes. (c) Surface plasmon absorption spectra of gold nanoparticles immersed into ethyl alcohol.

cesses were shown in Fig. 3a. The particle diameter and size distribution of nanoparticles was estimated to be $16.64 \pm 2.90 \mathrm{~nm}$. After selective growth processes, the particle diameter and size distribution of nanoparticles was estimated to be $16.71 \pm 3.21 \mathrm{~nm}$ in Fig. 3b. We found that uniform size-distribution and small particle-size can be remained after selective growth processes.

Figure 2c and d show SEM images of gold nanoparticles selective growth by electron beam lithography. The pattern-size of Fig. $2 \mathrm{c}$ is $100 \mathrm{~nm}$, and there is $\sim 20$ gold nanoparticles in the pattern. The pattern-size of Fig. $2 \mathrm{~d}$ is $\sim 40 \mathrm{~nm}$, and there are only 6 nanoparticles in the pattern. If the resist pattern can be shrunk further, selective growth one particle would be possible by this method. The concept for selective growth of nanomaterials shown in Fig. 1a is similar to the lift-off method in semiconductor processes. In general the lift-off method is difficult for sub-500 nm processes. However, this method can be used for patterning sub-50 nm nanomaterials. Nanoparticles can link to the APTMS layer and cannot be linked to the resist surface that is different to the conventional lift-off method.

After resist patterning, it is important to find a suitable solvent to strip the residual resists that would not affect selective growth of nanomaterials. In this paper, ethyl alcohol was used to strip the residual resists. By measuring the surface plasmon resonance absorption spectra of gold nanoparticles, the size and shape of nanoparticles can be realized. ${ }^{15}$ Figure $3 \mathrm{c}$ shows the absorption spectra of gold nanoparticles immersed into ethyl alcohol with different duration. The surface plasmon resonance spectra are unchanged with different immersion time and the peaks are found around $520 \mathrm{~nm}$ before and after resist strip processes. Therefore, the size and shape of nanoparticles can be remained by using ethyl alcohol to strip resists.

Similarly, sulfuric acid was used to strip resist. Figure 4 a shows the absorption spectra of gold nanoparticles immersed into sulfuric acid solution with different duration. The surface plasmon resonance absorption bands are becoming weaker and broader as longer immersion time and the peaks are shift to longer wavelengths. This means that the particle-size and size-deviation are increased. ${ }^{16}$ Figure $4 \mathrm{~b}-\mathrm{d}$ show the SEM images of gold nanoparticles immersed into sulfuric acid solution with 1, 5, and 25 min, respectively. We found that the size-deviation and shape of the nanoparticles were seriously affected by sulfuric acid. This is due to the citrate stabilizer covered on gold nanoparticles was desorbed by sulfuric acid. Therefore, sulfuric acid is not suitable for resist strip processes. 


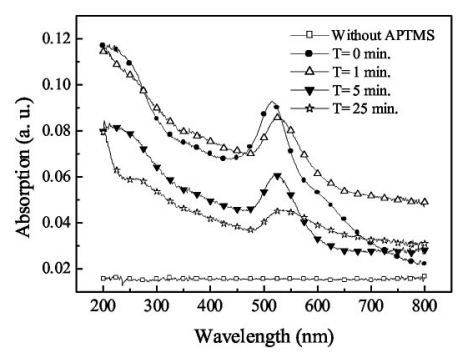

(a)

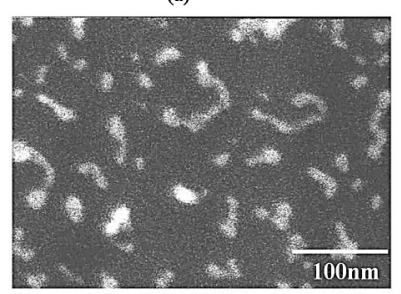

(c)

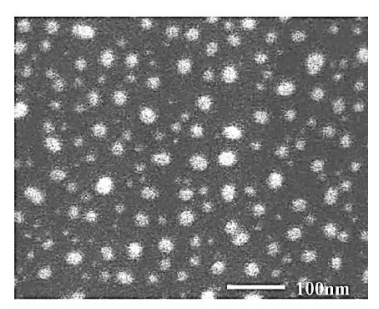

(b)

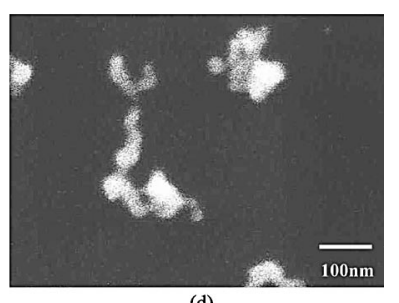

(d)
Figure 4. (a) Surface plasmon absorption spectra of gold nanoparticles immersed in sulfuric acid solution. SEM images of gold nanoparticles immersed into sulfuric acid solution with (b) 1, (c) 5, and (d) $25 \mathrm{~min}$.

\section{Conclusions}

Rapidly and precisely selective growth of self-assembled nanoparticles by using an electron-beam or optical exposure system with commercial chemically amplified resists was demonstrated. For using chemically amplified resists, the required exposure dosage was less than $10 \mu \mathrm{C} / \mathrm{cm}^{2}$. After the patterned resist sampel was immersed into the nanoparticles colloidal solution, nanoparticles were self-assembled on the APTMS layer. Suitable solvent such as ethyl alcohol was used to strip the residual resists. The surface plasmon resonance spectra were unchanged before and after resist strip processes. Therefore, the size and shape of nanoparticles can be maintained using ethyl alcohol to strip resists. The selective growth area of nanoparticles can be formed from several hundred micrometers to sub-50 nm. Nanoparticles can be selectively linked on the APTMS layer and cannot be linked on the resist surface, which is different to the conventional lift-off method. Therefore, this method can be used for selective growth of nanomaterials in sub-50 nm area. This method also has great potential to be used for selective growth of various nanoparticles or nanomaterials and is suitable to combine with semiconductor processes for mass production.

\section{Acknowledgments}

The authors are thankful to the National Science Council, Taiwan, R.O.C. for support in project NSC 93-2216-E-492-001.

National Taiwan University assisted in meeting the publication costs of this article.

\section{References}

1. T. Teranishi, I. Kiyokawa, and M. Miyake, Adv. Mater. (Weinheim, Ger.), 10, 596 (1998).

2. S. T. Liu, R. Maoz, G. Schmid, and J. Sagiv, Nano Lett., 2, 1055 (2002).

3. J. W. Zheng, Z. H. Zhu, H. F. Chen, and Z. F. Liu, Langmuir, 16, 4409 (2000).

4. S. Hoeppener, R. Maoz, S. R. Cohen, L. Chi, H. Fuchs, and J. Sagiv, Adv. Mater. (Weinheim, Ger.), 14, 1036 (2002).

5. R. Maoz, E. Frydman, S. R. Cohen, and J. Sagiv, Adv. Mater. (Weinheim, Ger.), 12, 725 (2000).

6. X. M. Lin, R. Parthasarathy, and H. M. Jaeger, Appl. Phys. Lett., 26, 1915 (2001).

7. R. Krupke, S. Malik, H. B. Weber, and O. Hampe, Nano Lett., 2, 1161 (2002).

8. C. K. Harnett, K. M. Satyalakshmi, and H. G. Craighead, Langmuir, 17, 178 (2001).

9. J. F. Liu, L. G. Zhang, N. Gu, J. Y. Ren, Y. P. Wu, Z. H. Lu, P. S. Mao, and D. Y. Chen, Thin Solid Films, 327, 176 (1998).

10. International Technology Roadmap for Semiconductor 2003, Semiconductor Industry Association (2003)

11. K. Suzuki, S. Matsui, and Y. Ochiai, Sub-Half-Micron Lithography for ULSI, Cambridge University Press, Cambridge (2000).

12. P. Rai-Choudhury, Handbook of Microlithography, Micromachining, and Microfab rication, SPIE Optical Engineering Press (1997)

13. P. C. Lee and D. J. Melsel, J. Phys. Chem., 86, 3391 (1982).

14. K. C. Grabar, K. J. Allison, B. E. Baker, R. M. Bright, K. R. Brown, R. G. Freeman, A. P. Fox, C. D. Keating, M. D. Musick, and M. J. Natan, Langmuir, 12, 2353 (1996).

15. A. C. C. Yu, M. Mizuno, Y. Sasaki, M. Inoue, and H. Kondo, Appl. Phys. Lett., 82, 2353 (2003).

16. S. Link and M. A. El-Sayed, J. Phys. Chem. B, 103, 4212 (1999). 